Chapter 19

\title{
Fiber Measurement Technique Based on OTDR
}

\author{
Masaharu Ohashi
}

Additional information is available at the end of the chapter

http://dx.doi.org/10.5772/54243

\section{Introduction}

An optical time domain reflect-meter (OTDR) has been developed for detecting the fault location and estimating the average loss of the installed fiber cables. The resolution of the distance has been improved up to a few centimeters. Recently, OTDR has been used for measurements of the transmission characteristics in fiber and optical fiber properties based on the backscattered signal power [1,2].

As a result of the rapid progress made in the field of optical fiber amplifiers (OFA's), it has become possible to construct long distance transmission systems without conventional 3R repeaters [3,4]. However, the use of OFA's gives rise to the problems of optical nonlinearities such as four-wave mixing (FWM), stimulated Brillouin scattering (SBS), and self-phase modulation (SPM). In OFA-based optical transmissions, both the chromatic dispersion distribution along a fiber and the average chromatic dispersion influence signal performance. Therefore, it has become important to know the longitudinal chromatic dispersion distribution for sophisticated transmission systems such as wavelength multiplexed and optical soliton transmission systems. However, conventional measurement techniques [5, 6] can only determine the average chromatic dispersion of either a short or a long fiber. Recently, there have been some reports on measurement techniques for estimating chromatic dispersion distribution along a fiber [7-9]. As these measurement techniques are based on optical nonlinear effects, the measurement distance is limited. On the other hand, we have reported the principle of a technique based on bidirectional measurement with OTDR [1].

In this chapter, we describe the measurement techniques for the longitudinal fiber parameters or transmission characteristics along the fiber. The backscattered power contains information on the fiber parameters at the scattered position such as mode field diame- 
ter, refractive index, and relative-index difference. Such information can be obtained by extracting the capture fraction from the backscattered power.

There are two types of techniques based on OTDR for measuring the longitudinal fiber properties by analyzing the backscattered power. One is the way of extracting the required information on the parameter from the capture fraction in the backscattered power just as it is. This technique is called "indirect method" in this section. The other is the way of adding the information into the backscattered power by utilizing the phenomenon between the signal and pump lights and required information can be easily obtained from the additional information in the backscattered power. This method is called "direct method". By using two types of techniques, the required information can be extracted from the backscattered power in the fiber.

In section 2, the principle of measurement technique for longitudinal fiber parameters such as the mode field diameter and relative-index difference and the reduction of polarization fluctuation influence in a fiber are described[2,10]. In section 3, the measurement technique for longitudinal transmission characteristics such as chromatic dispersion and the Raman gain efficiency are described. In this section, the direct and the indirect methods are described. In section 4, we summarize the measurement technique based on OTDR.

\section{Measurement technique for longitudinal fiber parameters}

This section describes the measurement techniques for longitudinal fiber parameters such as mode field diameter and relative-index difference.

\subsection{Mode field diameter distribution}

\subsubsection{Measurement principle}

The backscattered power $P(z)$ received from a given position $\mathrm{z}$ in a single-mode fiber can be expressed as $[2,11]$

$$
P(z)=P_{0} \alpha_{s}(z) B(z) \exp \left[-2 \int_{0}^{z} \gamma(x) d x\right]
$$

where $P_{0}$ is the input power, $\alpha_{\mathrm{s}}(\mathrm{z})$ the local scattering coefficient, $B(z)$ the backscattering capture fraction, and $\gamma(z)$ the local attenuation coefficient.

A reliable way to separate the effects of decay and waveguide imperfections from backscattered signals has already been described $[1,2,10]$. For OTDR signals $S_{1}(\lambda, z)$ and $S_{2}(\lambda, L-z)$ (in $\mathrm{dB}$ ) launched from opposite ends (subscripts 1 and 2) of a fiber of length $L$, the imperfection contribution $I(\lambda, z)$ can be expressed as [2] 


$$
\begin{aligned}
I(\lambda, z) & =\frac{S_{1}(\lambda, z)+S_{2}(\lambda, L-z)}{2} \\
& =a_{0}+10 \log \left[\alpha_{s}(z) B(\lambda, z)\right]-2(10 \log e) \int_{0}^{z} \gamma(x) d x
\end{aligned}
$$

where $\mathrm{a}_{0}$ is a constant independent of distance $\mathrm{z}$ and is expressed as

$$
a_{0}=5 \log \left(P_{0} P_{1}\right)-10(\log e) P_{0} \alpha_{s}(z) \int_{0}^{L} \gamma(x) d x
$$

Therefore, the imperfection contribution $I(\lambda, z)$ depends on the local scattering coefficient $\alpha_{\mathrm{s}}$ (z) and the backscattered capture fraction $B(\lambda, z)$, which is given by[12]

$$
B(\lambda, z)=\frac{3}{2}\left(\frac{\lambda}{2 \pi n(z) w(\lambda, z)}\right)^{2}
$$

where $n(z)$ and $2 w(\lambda, z)$ denote the refractive index of the core and the mode field diameter (MFD) at a wavelength of $\lambda$, respectively.

With conventional single-mode fibers, the variation in the local scattering coefficient $\alpha_{\mathrm{s}}(\mathrm{z})$ is negligible compared to that in the mode field diameter $2 w(\lambda, z)[13]$. Therefore, the imperfection contribution $I_{n}(\lambda, z)$ normalized by the value at a reference point $z=z_{0}$ is

$$
I_{n}(\lambda, z) \equiv I(\lambda, z)-I\left(\lambda, z_{0}\right)=20 \log \left[\frac{2 w\left(\lambda, z_{0}\right)}{2 w(\lambda, z)}\right]
$$

When the mode field diameter $2 w\left(\lambda, z_{0}\right)$ at $z=z_{0}$ is given, the mode field diameter distribution $2 w(\lambda, z)$ can be obtained as

$$
2 w(\lambda, z)=2 w\left(\lambda, z_{0}\right) 10^{-\frac{I_{n}(\lambda, z)}{20}}
$$

However, in a fiber link composed of different kinds of fiber, the local scattering coefficient $\alpha_{s}(z)$ and refractive index of the core $n(z)$ of each fiber should be taken into account because these values are different.

Here, we consider the measurement procedure for a fiber link composed of different types of fibers, by taking into account the difference between the scattering coefficients of the composed fibers. 
When the scattering coefficient and the refractive index change along the fiber are taken into account, the imperfection contribution $I(\lambda, z)$ can be expressed as

$$
\begin{gathered}
I(\lambda, z)=10 \log \left[\frac{\alpha_{s}(\lambda, z)}{n^{2}(z)}\right]+20 \log \left[\frac{1}{2 w(\lambda, z)}\right]+a_{1} \\
a_{1}=10 \log \left[\frac{3 \lambda^{2}}{8 \pi^{2}}\right]+a_{0}
\end{gathered}
$$

where $a_{1}$ is a constant independent of distance $z$. The imperfection contribution $I_{n}(\lambda, z)$ normalized by that at $\mathrm{z}=\mathrm{z}_{0}$ can be written from (7) as

$$
I_{n}(\lambda, z) \equiv I(\lambda, z)-I\left(\lambda, z_{0}\right)=10 \log \left[\frac{\alpha_{s}(z) n^{2}\left(z_{0}\right)}{\alpha_{s}\left(z_{0}\right) n^{2}(z)}\right]+20 \log \left[\frac{2 w\left(\lambda, z_{0}\right)}{2 w(\lambda, z)}\right]
$$

Here, we define the first term on the right hand side in (9) as a correction factor $K$. The local scattering coefficient $\alpha_{s}(z)$ is propotional to the Rayleigh scattering coefficient R. The Rayleigh scattering coefficient $\mathrm{R}$ for $\mathrm{GeO}_{2}$-doped core fiber is expressed as [14]

$$
R=R_{0}(1+0.62 \Delta)
$$

where $R_{0}$ and $\Delta$ denote the Rayleigh scattering coefficient of $\mathrm{SiO}_{2}$ glass and the relative-index difference in $\%$, respectively [14]. The refractive index $\mathrm{n}$ of the core can be expressed using the relative-index difference $\Delta$ as

$$
n=n_{0} / \sqrt{1-2 \Delta / 100}
$$

where $\mathrm{n}_{0}$ is the refractive index of the cladding. Using this relation, the correction factor $K$ is written as

$$
K=10 \log \left[\left\{\frac{1+0.62 \Delta(z)}{1+0.62 \Delta\left(z_{0}\right)}\right\}\left\{\frac{50-\Delta(z)}{50-\Delta\left(z_{0}\right)}\right\}\right]
$$

Therefore, the mode field diameter $2 \mathrm{w}(\lambda, \mathrm{z})$ distribution can be obtained as 


$$
2 w(\lambda, z)=2 w\left(\lambda, z_{0}\right) 10^{-\left\{\frac{I_{n}(\lambda, z)-K}{20}\right\}}
$$

On the contrary, another procedure has been proposed [15]. To circumvent this difficulty, a procedure similar to that introduced in [16], but with an easier implimentation is used. Like [16], two fitting coefficients $A(\lambda)$ and $C(\lambda)$ are introduced so that

$$
\begin{aligned}
I_{n}(\lambda, z) & \equiv I(\lambda, z)-I\left(\lambda, z_{0}\right) \\
& =20 A(\lambda) \log \left[\frac{2 w\left(\lambda, z_{0}\right)}{2 w(\lambda, z)}\right]+C(\lambda)
\end{aligned}
$$

Suppose to set a second reference point $z=z_{1}$. Two subscriptions can be made in (14), $z=z_{0}$ for the first reference point and $z=z_{1}$ for the second reference one. The resulting values for the coefficients $A(\lambda)$ and $C(\lambda)$ are

$$
A(\lambda)=\frac{I\left(\lambda, z_{1}\right)-I\left(\lambda, z_{0}\right)}{20 \log \frac{2 w\left(\lambda, z_{0}\right)}{2 w\left(\lambda, z_{1}\right)}}, \quad C(\lambda)=0
$$

From (14) and (15), the MFD becomes

$$
2 w(\lambda, z)=2 w\left(\lambda, z_{0}\right)\left[\frac{2 w\left(\lambda, z_{1}\right)}{2 w\left(\lambda, z_{0}\right)}\right]^{\frac{I(\lambda, z)-I\left(\lambda, z_{1}\right)}{I\left(\lambda, z_{0}\right)-I\left(\lambda, z_{1}\right)}}
$$

Thus, we need to know the MFD at the two reference points $z_{0}$ and $z_{1}$. From a experimental view point, two reference fibers have to be connected in front of the fiber link. This method is very effective when these fibers have different material characteristics.

\subsubsection{Reduction of polarization fluctuation influence in a fiber}

The polarization state of an optical pulse is continuously changing as the pulse propagates in a fiber, and this polarization fluctuation causes measurement error. This fluctuation must therefore be reduced during the backscattered power accumulation. It is believed that the polarization fluctuation can be reduced by switching the polarization state from 0 to 90 linear polarization during the measurement [2]. Figure 1 shows a block diagram of our experimental setup for measuring the backscattered power. 


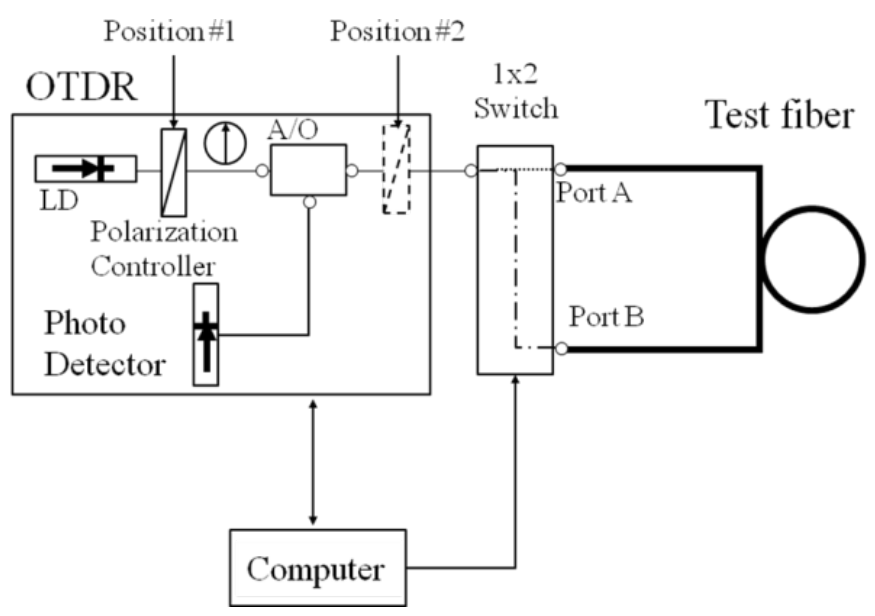

Figure 1. Block diagram of experimental setup

In our investigation, we refer to the positions of the polarization controller shown in Fig. 1 as positions \#1 and \#2. For simplicity, we defined the Jones matrix of the models shown in Fig. 1 as

$$
\mathbf{M}=\mathbf{R}(\theta) \mathbf{F R}(-\theta)
$$

where $\mathbf{R}$ is the rotation matrix and $\theta$ denotes the angle from the principal axis. The rotation matrix $\mathbf{R}$ is expressed as

$$
\mathbf{R}(\theta)=\left[\begin{array}{cc}
\cos \theta & -\sin \theta \\
\sin \theta & \cos \theta
\end{array}\right]
$$

$\mathbf{F}$ is the Jones matrix of the fiber which is expressed as

$$
\boldsymbol{F}=\left[\begin{array}{cc}
1 & 0 \\
0 & \exp (j \phi)
\end{array}\right]
$$

where $\phi$ is the birefringence of the fiber. Jones matrices for the detector through the $\mathrm{A} / \mathrm{O}$ switch $\mathbf{M}_{\mathrm{AO}}$, the polarization controller $\mathbf{P}_{\mathrm{c}}$ and input electric field vector $\mathbf{E}$ can be expressed as the following equations:

$$
\mathbf{M}_{A O}=\left[\begin{array}{cc}
m_{1} & 0 \\
0 & m_{2}
\end{array}\right]=m_{1}\left[\begin{array}{ll}
1 & 0 \\
0 & k
\end{array}\right]
$$




$$
\begin{gathered}
\mathbf{P}_{C_{x}}=\left[\begin{array}{ll}
1 & 0 \\
0 & 0
\end{array}\right], \quad \mathbf{P}_{C_{y}}=\left[\begin{array}{ll}
0 & 0 \\
0 & 1
\end{array}\right] \\
\mathbf{E}_{x}=\left[\begin{array}{l}
1 \\
0
\end{array}\right], \quad \mathbf{E}_{y}=\left[\begin{array}{l}
0 \\
1
\end{array}\right]
\end{gathered}
$$

When $x$-polarized light is launched into the fiber, the detected power $P_{x}$, shown in Fig. 1 as position \#1, is expressed as

$$
P_{x}=\left|\mathbf{M}_{A O} \mathbf{M E}_{x}\right|^{2}=m_{1}^{2}\left\{1+4\left(k^{2}-1\right) \sin ^{2} \theta \cos ^{2} \theta \sin ^{2}(\phi / 2)\right\}
$$

The detected power $P_{y}$ for -polarized light input is expressed as

$$
P_{y}=\left|\mathbf{M}_{A O} \mathbf{M E}_{y}\right|^{2}=m_{1}^{2}\left\{k^{2}-4\left(k^{2}-1\right) \sin ^{2} \theta \cos ^{2} \theta \sin ^{2}(\phi / 2)\right\}
$$

Using (23) and (24), the total detected power $P$ is expressed as the sum of the polarization components

$$
P=P_{y}+P_{y}=m_{1}^{2}\left(1+k^{2}\right)
$$

Equation (25) shows that the obtained power $P$ is independent of $\theta$ and $\phi$. The total detected power $P^{\sharp}$, shown in Fig. 1 as position \#2, is expressed as

$$
\begin{aligned}
P^{\#} & =P_{x}^{\#}+P_{y}^{\#}=\left|\mathbf{M}_{A O} \mathbf{P}_{C x} \mathbf{M E}_{x}\right|^{2}+\left|\mathbf{M}_{A O} \mathbf{P}_{C y} \mathbf{M E}_{y}\right|^{2} \\
& =m_{1}^{2}\left(1+k^{2}\right)\left\{1-4 k^{2} \sin ^{2} \theta \cos ^{2} \theta \sin ^{2}(\phi / 2)\right\}
\end{aligned}
$$

From (26), it is found that the obtained power depends on $\theta$ and $\phi$. Therefore, the polarization controller must be located between the LD and the A/O switch, shown in Fig. 1 as position \#1.

By using the polarization controller, the backscattered power can be suppressed due to the polarization fluctuation. 


\subsubsection{Experimental results}

To confirm the present technique, the mode field diameter distribution along the fiber link composed of the three fibers was measured. OTDR was used to measure the backscattered power of the fiber link. Table I summarizes the fiber parameters in the fiber link.

\begin{tabular}{cccc}
\hline Parameters & Fiber A & Fiber B & Fiber C \\
\hline MFD $(\mu \mathrm{m})$ at 1310nm & 9.02 & 6.47 & 6.49 \\
at 1550nm & 10.17 & 7.86 & 7.86 \\
\hline Cutoff wavelength $\lambda_{c}(\mathrm{~nm})$ & 1260 & 1150 & 1010 \\
\hline Relative-index difference $\Delta(\%)$ & 0.37 & 0.78 & 0.77 \\
\hline Chromatic dispersion $D(\mathrm{ps} / \mathrm{km} / \mathrm{nm})$ at $1550 \mathrm{~nm}$ & 16.72 & -0.29 & -0.27 \\
\hline Fiber length $(\mathrm{km})$ & 25.0 & 9.99 & 20.19 \\
\hline
\end{tabular}

Table 1. Parameters of test fibers

Figure 2 shows the bi-directional OTDR traces of the fiber link at $\lambda=1550 \mathrm{~nm}$. OTDR (Agilent E6003) with wavelengths of 1550 and $1310 \mathrm{~nm}$ was used to measure the backscattered signal powers for the fiber link. In our measurements, the pulse width of the OTDR was $1 \mu$ s and the averaging time was 10 minutes.

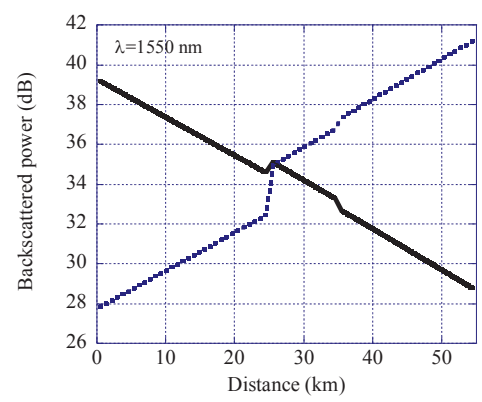

Figure 2. Bi-directional OTDR traces at $\lambda=1550 \mathrm{~nm}$

It is found that three fibers were spliced in the fiber link. The imperfection loss obtained from (2) was shown by using the Fig.1. Figure 3 shows the inperfection loss of the fiber link.

It is found that the losses of Fibers A, B, and C fluctuate along the fiber length. This fluctuation has an effect on the mode field diameter of each fiber. The MFD distribution at $\lambda=1550$ nm using Fig. 3 is shown in Fig. 4.

The MFD was estimated by using double reference method. When we measure the MFD distribution of the fiber link, Fibers A and B was used as reference fibers. The MFD was esti- 
mated by using (16) and MFDs at the double reference points. The deviations of the MFD for Fiber A, B and C were $0.039 \mu \mathrm{m}, 0.023 \mu \mathrm{m}$, and $0.046 \mu \mathrm{m}$, respectively. It is clarified that the longitudinal MFD deviation is negligible small for the fibers fabricated by the present fabrication technique.

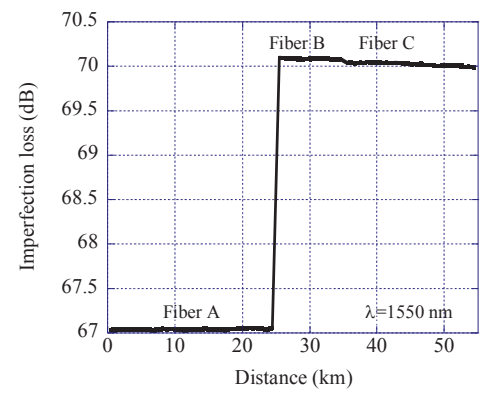

Figure 3. Imperfection loss of the fiber link

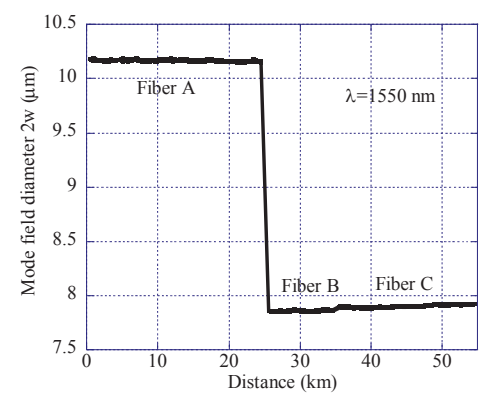

Figure 4. MFD distribution of the fiber link at $\lambda=1550 \mathrm{~nm}$

\subsection{Relative-index difference distribution}

\subsubsection{Measurement principle}

The first term on the right hand side in (9) depends on the variations in the scattering coefficient and refractive index of the core. The local scattering coefficient is proportional to the Rayleigh scattering coefficient. The Rayleigh scattering coefficient $\mathrm{R}$ for $\mathrm{GeO}_{2}$ doped core fiber is expressed as [14]

$$
R=R_{0}(1+k \Delta)
$$


where $R_{0}$ and $\Delta$ denote the Rayleigh scattering coefficient for $\mathrm{SiO}_{2}$ and the relative index difference $\Delta$ in $\%$, respectively. The $k$ value was estimated experimentally to be 0.62 in [14].

As the variation in the refractive-index $n(z)$ of the core along the fiber link is negligible, the following equation holds very well even if the fiber link is composed of different kinds of fiber[10].

$$
n^{2}\left(z_{0}\right) / n^{2}(z) \cong 1
$$

Thus, (9) can be rewritten as by using (27) and (28).

$$
\begin{aligned}
I_{n}(\lambda, z) & \equiv I(\lambda, z)-I\left(\lambda, z_{0}\right) \\
& \cong 10 \log \left[\frac{\alpha_{s}(z)}{\alpha_{s}\left(z_{0}\right)}\right]+20 \log \left[\frac{2 w\left(\lambda, z_{0}\right)}{2 w(\lambda, z)}\right] \\
& =10 \log \left[\frac{1+k \Delta(z)}{1+k \Delta\left(z_{0}\right)}\right]+20 \log \left[\frac{2 w\left(\lambda, z_{0}\right)}{2 w(\lambda, z)}\right]
\end{aligned}
$$

Here, if the MFD distribution and the relative index difference $\Delta\left(z_{0}\right)$ are known, the relative index difference $\Delta(z)$ can be derived from (29) as follows[10].

$$
\Delta(z)=\frac{1}{k}\left[\left(1+k \Delta\left(z_{0}\right) 10 \frac{I_{n}(\lambda, z)-20 \log \left[\frac{2 w\left(\lambda, z_{0}\right)}{2 w(\lambda, z)}\right]}{10}-1\right]\right.
$$

The MFD distribution along the transmission line can be easily estimated by using the double reference method [15]. With this technique, the imperfection loss of each test fiber along the transmission line can be estimated by comparing it with the imperfection losses at two reference points.

Next, the principal estimation error of the proposed method is discussed. The $\Delta$ estimation error was calculated by using (9) and (30) and can be written as

$$
\Delta_{E}(z)-\Delta_{R}(z)=\frac{1}{k}\left[\left\{\left(\frac{n^{2}\left(z_{0}\right)}{n^{2}(z)}\right)-1\right\}\left(1-k \Delta_{R}(z)\right)\right]
$$

where $\Delta_{R}$ and $\Delta_{E}$ denote the correct and estimated $\Delta$ values, respectively. From (31) we find that the $\Delta$ estimation error is directly proportional to $\left[n^{2}\left(z_{0}\right) / n^{2}(z)-1\right]$. 
Figure 5 shows the relationship between the correct relative index difference $\Delta_{\mathrm{R}}$ and the relative $\Delta$ estimation error (\%) against the refractive index at the $0.35 \%$ and $0.8 \%$ reference points. Here, a $\mathrm{k}$ value of 0.62 was used. It is seen that the relative $\Delta$ estimation error increases as the relative index difference between the test fiber and the reference fiber increases. Therefore, a reference fiber should be selected that has almost the same refractive index as that of the test fiber in order to estimate the relative index difference accurately.

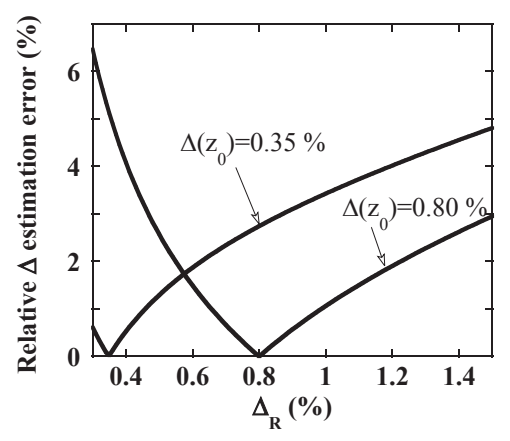

Figure 5. Relationship between the correct index difference $\Delta_{R}$ and relative $\Delta$ estimation error against refractive index as reference points of $0.35 \%$ and $0.8 \%$

\subsection{Experimental results}

To confirm the effectiveness of the present method, I measured the relative index difference distribution $\Delta(z)$ along a fiber link composed of one single-mode fiber (Fiber A) and two dispersion-shifted fibers (Fibers B and C). The parameters of these test fibers are listed in Table 1.

OTDR (Agilent E6003) with wavelengths 1550 and $1310 \mathrm{~nm}$ was used to measure the backscattered signal powers for the fiber link. In our measurements, the pulse width of the OTDR was $1 \mu$ s and the averaging time was 10 minutes. The spatial resolution of the relative index difference $\Delta$ estimation depends on the OTDR pulse width. In the experiments, the spatial resolution was about $200 \mathrm{~m}$.

Figure 6 shows the relative index difference distribution $\Delta(\mathrm{z})$ in the fiber link by using the MFD distribution as shown in Fig. 4. $k=0.62$ was used in (17). The $\Delta$ distribution of Fibers $\mathrm{B}$ and $\mathrm{C}$ are also shown in Fig. 6. It is found that the $\Delta(\mathrm{z})$ of Fiber $\mathrm{C}$ decreases slightly along the fiber length. The $\Delta$ variation of Fiber $C$ was estimated to be $0.01 \%$ from Fig. 6. In addition, the experimental results for the relative index difference obtained with the present method are in good agreement with the values measured with refractive near field (RNF) method.

This study proposed a novel relative-index difference distribution measurement method for a fiber link based on the use of an OTDR. It was clarified experimentally that the method can be applied to fiber links composed of different kinds of fiber. The relative-index differ- 
ence is one of important parameters to estimate the chromatic dispersion. However, there have been no reports on the technique for estimating the relative-index difference distribution. As far as we know, for the first time, we proposed the novel technique for estimating the relative-index difference distribution of the fiber link based on the OTDR.

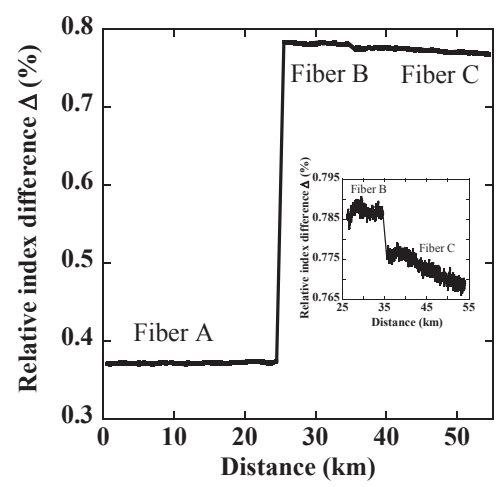

Figure 6. Relative-index difference distribution $\Delta(z)$ in the fiber link

\section{Measurement technique for longitudinal transmission characteristics}

This section describes the measurement techniques for longitudinal transmission characteristics such as chromatic dispersion and Raman gain efficiency and the experimental results.

\subsection{Chromatic dispersion distribution}

\subsubsection{Measurement principle}

The chromatic dispersion is expressed as the sum of the material dispersion $D_{m}$ and the waveguide dispersion $D_{w}$.

$$
D=D_{m}+D_{w}
$$

Here, $D_{m}$ and $D_{w}[17]$ are expressed as

$$
D_{m}=-\frac{\lambda}{c} \frac{d^{2} n}{d \lambda^{2}}
$$




$$
D_{w}=\frac{\lambda}{2 \pi^{2} c n} \frac{d}{d \lambda}\left(\frac{\lambda}{w^{2}}\right)
$$

where $\mathrm{c}$ is the light velocity. The material dispersion can be estimated from the dopant concentration in an optical fiber by using Sellmeier's coefficients [18]. The dopant concentration can be obtained from the relative-index difference $\Delta$ of the core. By contrast, the waveguide dispersion $D_{w}$ can be estimated by determining the wavelength dependence of the mode field diameter.

The empirical relationship between the mode-field diameter $2 w$ and the normalized frequency $v$ has been reported by Marcuse as [19]

$$
\frac{w}{a}=b_{0}+b_{1} v^{-1.5}+b_{2} v^{-6}=c_{0}+c_{1}\left(\frac{\lambda_{c}}{\lambda}\right)^{-1.5}+c_{2}\left(\frac{\lambda_{c}}{\lambda}\right)^{-6}
$$

where $2 a$ is the core diameter and $\lambda_{c}$ the cutoff wavelength.

Here, we approximated the wavelength dependence of the mode-field diameter(MFD) $2 w$ as

$$
w(\lambda, z)=g_{0}(z)+g_{1}(z) \lambda^{1.5}+g_{2}(z) \lambda^{6}
$$

Substituting (36) into (34), the waveguide dispersion can be expressed as

$$
D_{w}=\frac{\lambda}{2 \pi^{2} c n w^{2}(\lambda, z)}\left\{1-\frac{2 \lambda}{w(\lambda, z)}\left(\frac{3}{2} g_{1}(z) \lambda^{0.5}+6 g_{2}(z) \lambda^{5}\right)\right\}
$$

Therefore, the waveguide dispersion $D_{w}(z)$ at the position $\mathrm{z}$ can be evaluated from the coeffcients $g_{0}(z), g_{1}(z)$ and $g_{2}(z)$. To obtain the coefficients $g_{0}(z), g_{1}(z)$ and $g_{2}(z)$, the MFD has to be measured at more than three wavelengths.

In general, as the coefficient of $g_{2}$ is negligible small compared with that of $g_{1}$, the wavelength dependence of the MFD can be expressed as

$$
w(\lambda, z)=g_{0}(z)+g_{1}(z) \lambda^{1.5}
$$

In this case, the coefficients $g_{0}$ and $g_{1}$ can be estimated by using the MFDs at the two wavelengths. The two wavelengths of 1.31 and $1.55 \mu \mathrm{m}$ are usually used for these measurements, which are the operating wavelengths in the current transmission systems.

Substituting (38) into (34), the waveguide dispersion can be expressed as 


$$
D_{w}=\frac{\lambda}{2 \pi^{2} c n w^{2}(\lambda, z)}\left\{1-\frac{2 \lambda}{w(\lambda, z)}\left(\frac{3}{2} g_{1}(z) \lambda^{0.5}\right)\right\}
$$

Here, we assume that the MFDs at the two wavelength $\lambda_{1}$ and $\lambda_{2}$ are respective $2 w\left(\lambda_{1}\right)$ and $2 w\left(\lambda_{2}\right)$. In this case, the coefficients $g_{0}$ and $g_{1}$ which represent the wavelength dependence of the MFD can be obtained as

$$
\begin{gathered}
g_{0}=\frac{w\left(\lambda_{2}\right) \lambda_{1}^{1.5}-w\left(\lambda_{1}\right) \lambda_{2}^{1.5}}{\lambda_{1}^{1.5}-\lambda_{2}^{1.5}} \\
g_{1}=\frac{w\left(\lambda_{1}\right)-w\left(\lambda_{2}\right)}{\lambda_{1}^{1.5}-\lambda_{2}^{1.5}}
\end{gathered}
$$

The chromatic dispersion can be measured by using the wavelength dependence of the MFD and the relative-index difference of the core. Both parameters MFD and the relativeindex difference can be easily estimated by using the OTDR. The MFD $2 w(z)$ and the relative-index difference $\Delta(z)$ can be measured by the technique as mentioned in section 2 .

\subsubsection{Experimental results}

The chromatic dispersion measurements were made on the fiber link composed of three different fibers. The parameters of these test fibers are listed in Table 1. The chromatic dispersion is sum of waveguide and material dispersion. The material dispersion can be estimated from the dopant concentration corrssponding to the relative-index difference. On the contrast, the waveguide dispersion can be obtained by using the wavelength dependence of MFD. The fiber link was measured by bi-directional OTDR with both wavelengths of 1310 and $1550 \mathrm{~nm}$. Figs. 4 and 7 show the MFD distributions at $\lambda=1550 \mathrm{~nm}$ and $1310 \mathrm{~nm}$. estimated by using the bi-directional OTDR traces, respectively. To estimate the MFDs, Fibers A and $\mathrm{B}$ were used as the reference fibers[15].

The waveguide dispersion can be estimated by using the MFD distributions at both 1310 and $1550 \mathrm{~nm}$ and (30). Figure 8 shows the waveguide dispersion of the fiber link at $\lambda=1550 \mathrm{~nm}$.

It is found that the waveguide dispersion of Fiber A (conventional single-mode fiber) was smaller than that of Fiber B and C (dispersion-shifted fibers). This is because the zero-dispersion wavelength can be shifted to the longer wavelength. On the other hand, the material dispersion can be calculated by using Sellmeire equation when the relative-index diffence is known. The relative-index differnce was estimated by using the technique presented in 2.2. Figure 9 shows the material dispersion of the fiber link at $\lambda=1550 \mathrm{~nm}$. 


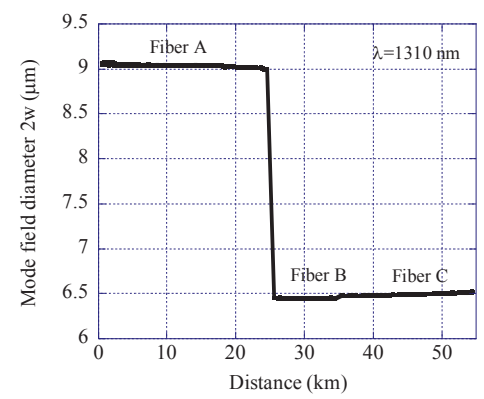

Figure 7. MFD distribution of the fiber link at $\lambda=1310 \mathrm{~nm}$

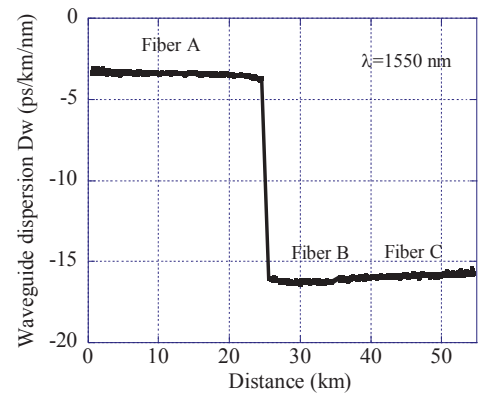

Figure 8. Waveguide dispersion of the fiber link at $\lambda=1550 \mathrm{~nm}$

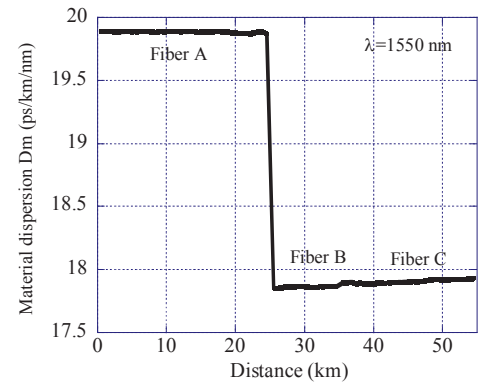

Figure 9. Material dispersion of the fiber link at $\lambda=1550 \mathrm{~nm}$

The chromatic dispersion at $\lambda=1550 \mathrm{~nm}$ can be estimated by using Figs 9 and 10. Figure 10 shows the chromatic dispersion of the fiber link at $\lambda=1550 \mathrm{~nm}$. 
We described a nondestructive technique for measuring the chromatic dispersion distribution along a single-mode fiber based on bidirectional OTDR measurements. This technique was compared with the destructive interferometric technique and found to be in good agreement. We also proposed a measurement procedure for a transmission line composed of different types of single-mode fibers. We confirmed experimentally that our technique can be applied to a transmission line. Our technique for estimating chromatic dispersion distribution will be a powerful tool for designing WDM and FDM transmission systems.

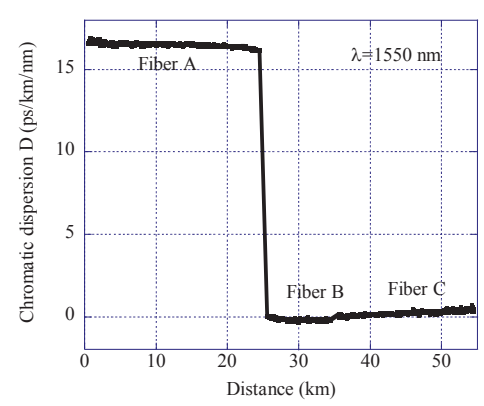

Figure 10. Chromatic dispersion distribution of the fiber link at $\lambda=1550 \mathrm{~nm}$

\subsection{Raman gain efficiency distribution}

The development of various kinds of Internet services has led to a rapidly increase in transmission capacity. With a view to realizing ultra-wide band transmission systems, wavelength division multiplexing (WDM) systems have been introduced together with Raman amplification technology [20]. Raman amplification technology is an attractive technology whereby the amplification wavelength region can be adjusted by changing the wavelength of the pump light wavelength. Technologies have been reported for measuring the Raman gain efficiency distribution using pump and signal lasers [21]. In this section, two types of techniques for measuring the Raman gain efficiency is described based on the OTDR. The first one is the direct method using pump lasers. The other is the in direct method without pump lasers.

\subsubsection{Measurement principle}

a. Direct method

Figure 11 shows the schematic diagram for measuring the Raman gain efficiency distribution in the optical fibers by using an OTDR. 


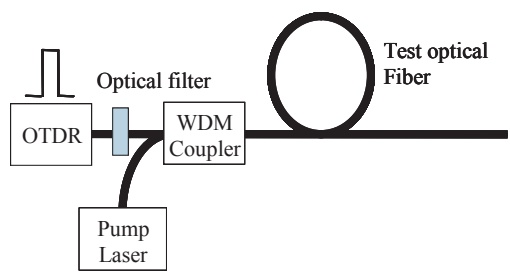

Figure 11. Schematic diagram of the proposed Raman gain efficiency measurement method

The pulsed signal and the continuous wave pump lights are launched into the test fiber through the WDM coupler. These lights co-propagate through the test optical fiber. An optical filter is inserted between the OTDR and the WDM coupler to eliminate the Rayleigh backscattering of the pump light.

Here, we derive the signal power at the distance of $z$. The signal power $P_{s}$ can be obtained by solving the coupled power equation with regard to the signal $P_{s}$ and the pump powers $P_{p}$. If the pump is un-depleted, the pump power can be expressed by the following equations [22].

$$
\begin{gathered}
\frac{d P_{s}}{d z}=\frac{g_{R}}{A_{e f f}} P_{p} P_{s}-\alpha_{s} P_{s} \\
\frac{d P_{p}}{d z}=-\alpha_{p} P_{p^{\prime}}
\end{gathered}
$$

where $g_{R}$ is the Raman gain coefficient. $\alpha_{s}$ and $\alpha_{p}$ are the attenuation coefficients of signal and pump wavelengths, respectively. $A_{\text {eff }}$ denotes the effective area, which corresponds to the overlapping area between pump and signal lights and is defined as

$$
A_{e f f}=2 \pi \frac{\int \phi_{s}^{2}(r) r d r \int \phi_{p}^{2}(r) r d r}{\int \phi_{s}^{2}(r) \phi_{p}^{2}(r) r d r}
$$

where $\phi_{s}$ and $\phi_{p}$ are the field distributions of the fundamental mode of the fiber at radius $\mathrm{r}$ at the respecitive signal and pump wavelengths.

In particular, when the field distribution is Gaussian, the effective area $A_{e f f}$ is obtained as

$$
A_{e f f} \cong \pi\left(w_{s}^{2}+w_{p}^{2}\right) / 2
$$


where $w_{s}$ and $w_{p}$ denote the mode field radii of the signal and pump wavelengths, respectively.

From (43), the pump power $P_{p}$ at the position of $\mathrm{z}$ can be obtained as

$$
P_{p}(z)=P_{p}(0) \exp \left(-\alpha_{p} z\right)
$$

Substituting (46) into (42), the signal power $P_{s}(z)$ at the position of $z$ can be obtained as

$$
P_{s}(z)=P_{s}(0) \exp \left[\int_{0}^{z}\left(\frac{g_{R} P_{p}(0)}{A_{e f f}} \exp \left(-\alpha_{p} z\right)-\alpha_{s}\right) d z\right]
$$

The signal power $P_{s}(z)$ at the position of $z$ is reflected and it travels toward the input direction. The backscattered light can be expressed as the product of the signal power $P_{s}(z)$ and $B(z) \alpha$, where $\alpha$ is the scattering coefficient and $B(z)$ is the backscatterd capture fraction. Then, the backscattered signal light $P_{s}(z) B(z) \alpha$ is amplified by the counter propagating pump light. Therefore, the backscattered power $P\left(z, P_{p}\right)$ from the position of $z$ can be expressed as

$$
\begin{aligned}
P\left(z, P_{p}\right) & =P_{s}(0) \alpha B(z) \exp \left[2 \int_{0}^{z}\left(\frac{g_{R} P_{p}(0)}{A_{e f f}} \exp \left(-\alpha_{p} z\right)-\alpha_{s}\right) d z\right] \\
= & P_{s}(0) \alpha B(z) \exp \left[2 P_{p}(0) G(z)\right] \times \exp \left[-2 \alpha_{s} z\right]
\end{aligned}
$$

where $G(z)$ is defined as

$$
G(z)=\int_{0}^{z} \frac{g_{R}(z)}{A_{e f f}(z)} \exp \left(-\alpha_{p} z\right) d z
$$

On the contrary, when the pump light is off, the backscattered power $P(z, 0)$ can be obtained by substituting $P_{p}(0)=0$ into (48) as

$$
P\left(z, P_{p}\right)=P_{s}(0) \alpha B(z) \exp \left[-2 \alpha_{s} z\right]
$$

The backscattered power of OTDR, $S\left(z, P_{p}\right)\left[=10 \log \left\{P\left(z, P_{p}\right)\right\}\right]$ can be expressed as 


$$
\begin{aligned}
S\left(z, P_{p}\right)= & 10 \log \left[P_{s}\left(z, P_{p}\right)\right]=10 \log \left[P_{s}(0)\right]+10 \log [\alpha B(z)] \\
& +2 P_{p}(0) G(z) \cdot 10 \log (e)-2 \alpha_{s} z 10 \log (e)
\end{aligned}
$$

The backscattered power at $z=z+\Delta z$ can be also expressed as

$$
\begin{aligned}
S\left(z+\Delta z, P_{p}\right) & =10 \log \left[P_{s}\left(z+\Delta z, P_{p}\right)\right] \\
& =10 \log \left[P_{s}(0)\right]+10 \log [\alpha B(z+\Delta z)] \\
& +2 P_{p}(0) G(z+\Delta z) \cdot 10 \log (e) \\
& -2 \alpha_{s}(z+\Delta z) 10 \log (e)
\end{aligned}
$$

The following equation can be derived from (50) and (51).

$$
\frac{d S\left(z, P_{p}\right)}{d z}=\frac{d\{10 \log [\alpha B(z)]\}}{d z}+2 P_{p}(0) \cdot 10 \log (e) \frac{d G(z)}{d z}-2 \alpha_{s} 10 \log (e)
$$

On the contrary, when $P_{p}=0$, the following equation can be also derived in the same manner.

$$
\frac{d S(z, 0)}{d z}=\frac{d\{10 \log [\alpha B(z)]\}}{d z}-2 \alpha_{s} 10 \log (e)
$$

From the definition of $G, d G(z) / d z$ can be expressed as

$$
\frac{d G(z)}{d z}=\frac{g_{R}(z)}{A_{e f f}(z)} \exp \left(-\alpha_{p} z\right)
$$

Therefore, the Raman gain efficiency $g_{R}(z) / A_{e f f}(z)$ at the position of $z$ can be derived from (52) to (54) as

$$
\frac{g_{R}(z)}{A_{e f f}(z)}=\frac{d S_{d}(z)}{d z} \cdot \frac{1}{2 P_{p}(0) \cdot 10 \log (e) \exp \left(-\alpha_{p} z\right)}
$$

Here, $S_{d}(z)$ corresponding to the backscattered power difference between with and without pumping is defined as 


$$
S_{d}(z)=S\left(z, P_{p}\right)-S(z, 0)
$$

Therefore, the Raman gain efficiency distribution can be estimated from the length dependence of the pump power and the derivative of $S_{d}(z)$ with regard to the fiber length $z$. It is also found from (56) that the Raman gain efficiency distribution $g_{R}(z) / A_{e f f}(z)$ can be estimated by using the conventional OTDR.

b. Indirect method

The Raman gain coefficient $g_{R}$ of $\mathrm{GeO}_{2}$-doped core fiber can be expressed by the following equation [23, 24].

$$
g_{R}=g_{0}(1+80 \Delta) / \lambda_{p}
$$

where $\Delta$ is the relative-index difference in $\%$ and $g_{0}$ denotes the Raman gain coefficient of pure silica glass. $\lambda_{p}$ is the pump wavelength.

The Raman gain efficiency can be expressed as $g_{R} / A_{\text {eff. }}$ Thus, the Raman gain efficiency can be obtained by using (44) and (58) as

$$
\frac{g_{R}}{A_{\text {eff }}}=\frac{g_{0}(1+80 \Delta)}{\lambda_{p} A_{\text {eff }}}
$$

From (59), we can approximate the Raman gain coefficient by using the relative-index difference $\Delta$ and mode field diameter (MFD) $2 w$. In the fiber link, the MFD $2 w$ and relative-index difference $\Delta$ distributions can be estimated by using the bi-directional OTDR technique as described in section 2 .

If we know the Raman gain efficiency $\left(g_{R}\left(z_{0}\right) / A_{e f f}\left(z_{0}\right)\right)$ of the reference fiber at the position $z=z_{0}$, the Raman gain efficiency $\left(g_{R}(z) / A_{e f f}(z)\right)$ of the test fiber at the position $z$ can be estimated from (59) as

$$
\frac{g_{R}(z)}{A_{e f f}(z)}=\frac{g_{R}\left(z_{0}\right)}{A_{e f f}\left(z_{0}\right)} \frac{(1+80 \Delta(z))}{\left(w_{s}^{2}(z)+w_{p}^{2}(z)\right)} \frac{\left(w_{s}^{2}\left(z_{0}\right)+w_{p}^{2}\left(z_{0}\right)\right)}{\left(1+80 \Delta\left(z_{0}\right)\right)}
$$

Here, if we assume that the wavelength dependence of MFD is negligible, (60) can be approximated as 


$$
\frac{g_{R}(z)}{A_{\text {eff }}(z)}=\frac{g_{R}\left(z_{0}\right)}{A_{\text {eff }}\left(z_{0}\right)}\left[\frac{w_{s}^{2}\left(z_{0}\right)}{w_{s}^{2}(z)} \frac{1+80 \Delta(z)}{1+80 \Delta\left(z_{0}\right)}\right]
$$

The Raman gain efficiency can be estimated by measuring both the MFD and the relativeindex difference distributions of the test fiber. Thus, the Raman gain efficiency can be obtained indirectly by using OTDR without pump lasers. This technique is a relative measurement method, so the measurement accuracy depends on the Raman gain efficiency of the reference fiber.

\subsubsection{Experimental results}

a. Direct method

The Raman gain efficiencies for a conventional single-mode fiber with a length of $25 \mathrm{~km}$ and a fibre link installed in the field with a length of $11 \mathrm{~km}$ composed of 22 conventional singlemode fibers with a piece length of $500 \mathrm{~m}$ were measured to confirm the effectiveness of our technique.

Figure 12 shows the backscattered powers with and without pumping for the conventional single-mode fiber. OTDR (Anritsu) with a wavelength of $1550 \mathrm{~nm}$ was used to measure the backscattered power. The OTDR pulse width was $1 \mu$ s and the averaging time was 5 min. The pump laser with a wavelength of $1475 \mathrm{~nm}$ was used. It is found that the signal power is amplified by the pump power.

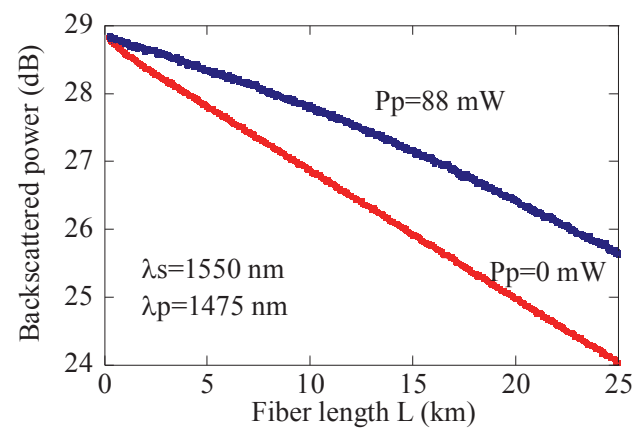

Figure 12. Backscattered powers with and without pumping

Figure 13 shows the backscattered power difference $S_{d}$ defined in (14) plotted as a function of fiber length. 


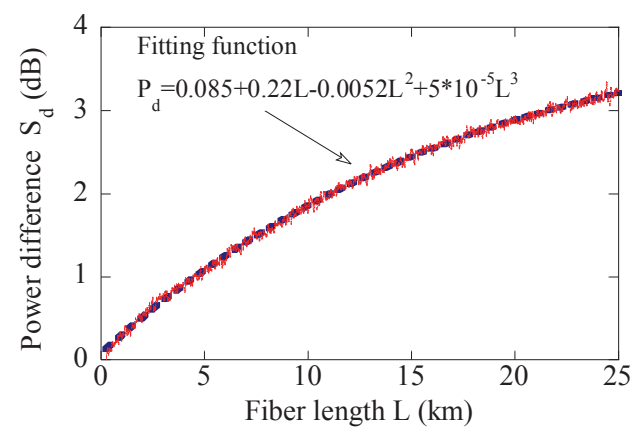

Figure 13. Backscattered power difference $S_{d}$

$\mathrm{S}_{\mathrm{d}}(\mathrm{z})$ was best fitted to the polynomial function as to calculate its derivative.

$$
S_{d}(z)=0.085+0.22 \cdot z-0.0052 \cdot z^{2}+5.0 \times 10^{-5} \cdot z^{3}
$$

The attenuation coefficient of the test fiber at $\lambda=1475 \mathrm{~nm}$ was $0.217 \mathrm{~dB} / \mathrm{km}$. The pump power $\mathrm{P}_{\mathrm{p}}(0)$ was $88 \mathrm{~mW}$. The Raman gain efficiency distribution along the fiber length can be estimated by using (56) and (62). Figure 14 shows the Raman gain efficiency distribution estimated by our technique.

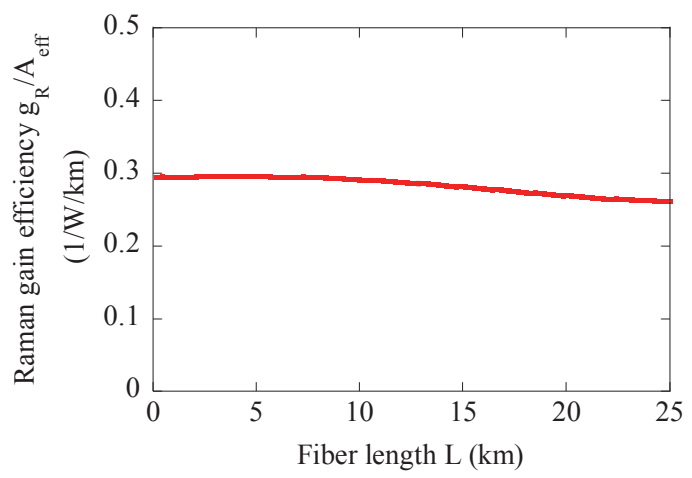

Figure 14. Raman gain efficiency distribution

It is seen that Raman gain efficiency along the fiber length is almost the same as $0.3 \mathrm{~W}^{-1} \mathrm{~km}^{-1}$.

Next, the Raman gain efficiency for the fiber link composed of 22 conventional single-mode fibers was measured. Figure 15 shows the backscattered powers with and without pumping and the backscattered power difference $S_{d}$ plotted as a function of fiber length. 


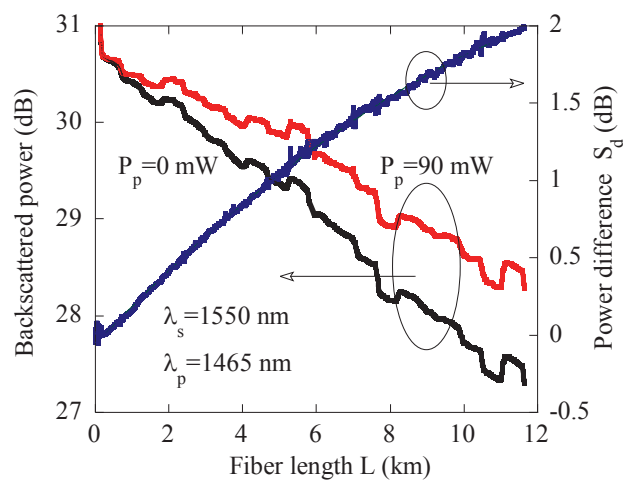

Figure 15. Backscattered powers with and without pumping and the backscattered power difference $S_{d}$ plotted as a function of fiber length

The attenuation coefficient $\alpha_{\mathrm{p}}$ was measured by the OTDR with a wavelength of $1450 \mathrm{~nm}$. The length dependence of the pump power $P_{p}(z)\left(=P_{p}(0) \exp \left(-\alpha_{p} z\right)\right)$ was estimated from the OTDR trace at the pump wavelength $\lambda_{\mathrm{p}}$. By using the length dependence of the pump power, and the derivative of $S_{d}$ with regard to the fiber length $\mathrm{z}$, the Raman gain efficiency distribution of the fiber link was estimated. The Raman gain efficiency of the fiber link is shown in Fig. 16.

It is found that the Raman gain efficiency distribution in the fiber link varies from 0.22 to $0.32 \mathrm{~W}^{-1} \mathrm{~km}^{-1}$. The Raman gain efficiency distribution of the fiber link installed in the field shows the appropriate value. As a result, it is confirmed that our technique can be applied to the fiber link.

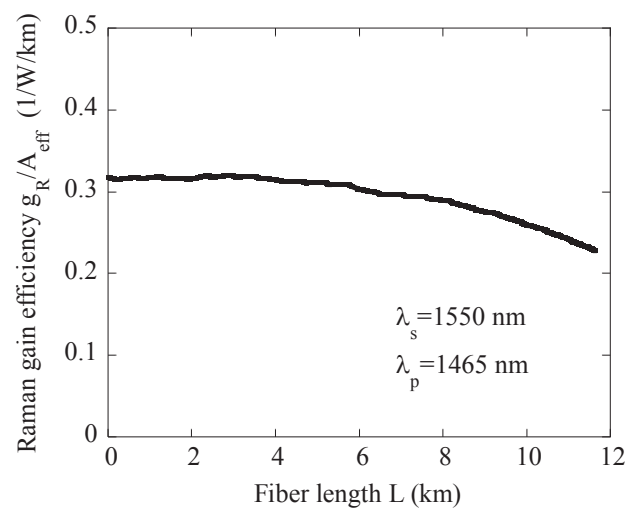

Figure 16. Raman gain efficiency of the fiber link 
b. Indirect method

The Raman gain efficiency of the concatenated fiber link as shown in Fig. 17 was measured to confirm the effectiveness of our technique.

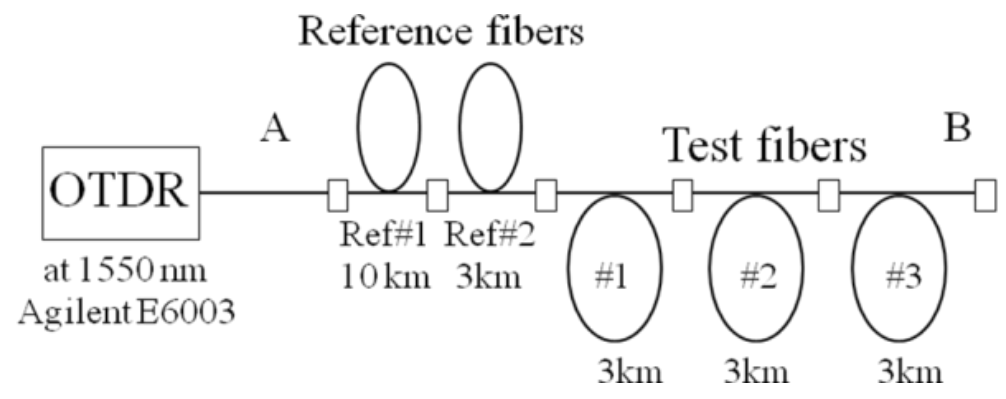

Figure 17. Concatenated fiber link

The fibers Ref\#1 and Ref\#2 were used as reference fibers for estimating the MFD and the relative-index difference $\Delta$ distributions in the concatenated fiber link. The parameters of these two reference fibers are listed in Table 2. Ref\#2, \#1, \#2 and \#3 are the conventional singlemode fibers. OTDR (Agilent E6003) was used to measure the backscattered signal powers for the concatenated fiber link.

\begin{tabular}{ccc}
\hline Parameters & Ref\#1 & Ref\#2 \\
\hline MFD $(\mu \mathrm{m})$ at 1550nm & 7.86 & 10.2 \\
\hline Cutoff wavelength $\lambda_{c}(\mathrm{~nm})$ & 1150 & 1130 \\
\hline Relative-index difference $\Delta(\%)$ & 0.78 & 3.0 \\
\hline Fiber length $L(\mathrm{~km})$ & 10.0 & 0.19 \\
\hline Loss $(\mathrm{dB} / \mathrm{km})$ & 0.20 & - \\
\hline
\end{tabular}

Table 2. Parameters of reference fibers

Figure 18 shows the MFD distribution at $\lambda=1550 \mathrm{~nm}$ in the concatenated fiber link estimated by [4]. In this measurement, the OTDR pulse width was $1 \mu$ s and the averaging time was 3 min. The parameters of test fibers $\# 1$ to $\# 3$ are listed in Table 3. 


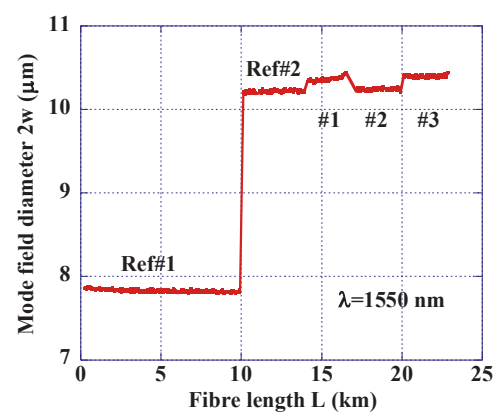

Figure 18. MFD distribution in the concatenated fiber link

\begin{tabular}{cccc}
\hline Parameters & \#1 & \#2 & \#3 \\
\hline MFD $(\mu \mathrm{m})$ at $1550 \mathrm{~nm}$ & 10.4 & 10.2 & 10.4 \\
\hline Cutoff wavelength $\lambda_{\mathrm{c}}(\mathrm{nm})$ & - & 1250 & - \\
\hline Fiber length L $(\mathrm{km})$ & 3.0 & 3.0 & 3.0 \\
\hline Loss $(\mathrm{dB} / \mathrm{km})$ at $1550 \mathrm{~nm}$ & 0.18 & 0.19 & 0.19 \\
\hline Raman gain efficiency $(1 / \mathrm{W} / \mathrm{km})$ & 0.66 & 0.75 & 0.64 \\
\hline
\end{tabular}

* The value measured by the direct technique.

Table 3. Parameters of test fibers

Figure 19 shows the relative-index difference $\Delta(z)$ distribution in the concatenated fiber link which was obtained by using the MFD distribution as shown in Fig. 19. It is seen that the relative-index differences $\Delta$ of $\operatorname{Ref} \# 2, \# 1, \# 2$, and $\# 3$ are almost the same and the $\Delta$ of the Ref\#1 is the largest among the test fibers.

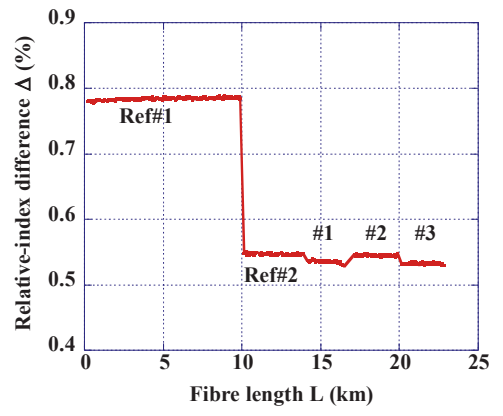

Figure 19. Relative-index difference $\Delta(z)$ distribution in the concatenated fiber link 
The Raman gain efficiency normalized by that of Ref\#2 was estimated from Figs. 18 and 19 by using (61), which is shown in Fig. 20. The Raman gain efficiency of Ref\#2 was estimated to be 0.75 by the direct measurement technique. It is seen that the Raman gain efficiency of Ref\# 1 is the largest among the test fibers because the relative-index difference $\Delta$ is large and the MFD is small. We found that the Raman gain efficiencies of Ref\#2 and $\# 2$ (group A) are larger than those of \#1 and \#3 (group B). Each group was fabricated by the same manufacturer. The ratio of the Raman gain efficiency of group $B$ fiber to that of group A fiber was about 1.1.

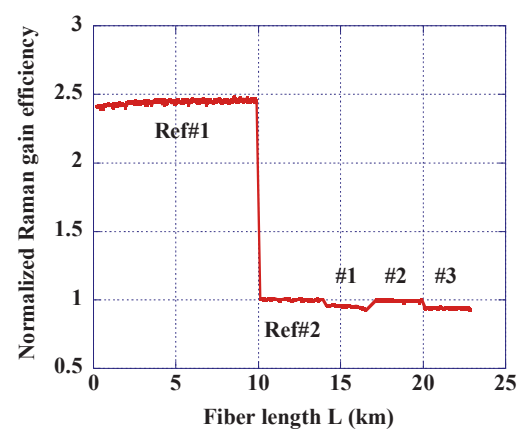

Figure 20. Normalized Raman gain efficiency in the concatenated fiber link

Next, we measured the Raman gain efficiency of the test fibers directly by using a pump laser. The experimental results are summarized in Table 3 . We found that each group fiber has almost the same the Raman gain efficiency. We also found that the ratio of the Raman gain efficiency of group B to that of group A is about 1.2, which is in good agreement with the results obtained with the present technique.

We described a new technique for measuring Raman gain efficiency distribution using a conventional OTDR. The Raman gain efficiency in the $25 \mathrm{~km}$ long single-mode fiber and the fiber link installed in the field with a length of $11 \mathrm{~km}$ composed of 22 conventional singlemode fibers were successfully estimated experimentally.

\section{Conclusion}

We described the measurement techniques for the longitudinal fiber parameters or transmission characteristics along the fiber based on the OTDR.

We described two types of techniques based on OTDR for measuring the longitudinal fiber properties by analyzing the backscattered power. One was the way of extracting the required information on the parameter from the capture fraction in the backscattered power just as it is. This technique is called "indirect method". As the backscattered capture fraction contains the information on the fiber parameters, the fiber parameters distibution can be ob- 
tained by analizing the backscattered capture fraction. The other was the way of adding the information into the backscattered power by utilizing the phenomenon between the signal and pump lights and required information can be easily from the additional power in the backscattered power. This method is called "direct method". By using two types of techniques, the required information can be extracted from the backscattered power in the fiber.

OTDR based measurement techniques will be powerful to estimate the various kinds of properties in the fibers or the optical transmission lines.

\section{Acknowledgements}

The author would like to express sincere thank to Prof. Tateda M. and Dr. Nakajima K. for their fruitful discussions. He would like to also thank Dr. Yamashita I., Dr. Tsutsumi Y. and Mr. Hatada H. for their helps during measurements.

\section{Author details}

Masaharu Ohashi

Address all correspondence to: ohashi@eis.osakafu-u.ac.jp

Osaka Prefecture University, Gakuen-cho, Naka, Sakai, Osaka, Japan

\section{References}

[1] Ohashi M. and Tateda M., "Novel technique for measuring longitudinal chromatic dispersion distribution in single-mode fibres," Electron Lett., vol. 29, pp. 426-427, 1993.

[2] Nakajima K., Ohashi M. and Tateda M., “Chromatic dispersion distribution measurement along a single-mode optical fiber," J. Lightwave Technol., vol. 15, pp. 1095-1101, 1997.

[3] Hagimoto K., Iwatsuki K., Takada A., Nakazawa M., Saruwatari M., Aida K., and Nakagawa K., “250 km nonrepeated transmission experiment at $1.8 \mathrm{~Gb} / \mathrm{s}$ using LD pumped $\mathrm{Er}_{3+}$-doped fiber amplifiers in IM/direct detection system," Electron. Lett., vol. 25, no. 10, pp. 662-664, 1989.

[4] Nakazawa M., Kimura Y., Suzuki K., Kubota H., Komukai T., Yamada E., Sugawa T., Yoshida E., Yamamoto T., Imai T., Sahara A., Nakazawa H., Yamauchi O., and Umezawa M., "Field demonstration of soliton transmission at $10 \mathrm{Gbit} / \mathrm{s}$ over $2000 \mathrm{~km}$ in 
Tokyo metropolitan optical loop network," Electron. Lett., vol. 31, no. 12, pp. 992-994, 1995.

[5] Tateda M., Shibata N., and Seikai S., "Interferometric method for chromatic dispersion measurement in a single-mode optical fiber," J. Quantum Electron., vol. QE-17, pp. 404-407, Mar. 1981.

[6] Daikoku K. and Sugimura A., "Direct measurement of wavelength dispersion in optical fibers - Difference method," Electron. Lett., vol.14, no. 5, pp. 149-151, 1978.

[7] Jopson R. M., Eiselt M., Stolen R. H., Derosier R. M., Vengsarkar A. M., and Koren U., "Non-destructive dispersion-zero measurements along an optical fiber," Electron. Lett., vol. 31, no. 24, pp. 2115-2117, 1995.

[8] Nishi S. and Saruwatari M., “Technique for measuring the distributed zero dispersion wavelength of optical fibers using pulse amplification caused by modulation instability," Electron. Lett., vol. 31, no. 3, pp. 225-226, 1995.

[9] Onaka H., Otsuka K., Miyata H., and Chikama T., "Measuring the longitudinal distribution of four-wave mixing efficiency in dispersionshifted fibers," Photon. Technol. Lett., vol. 6, pp. 1454-1456, Dec. 1994.

[10] Ohashi M.,"Novel OTDR technique for measuring relative-index difference of fiber links“, IEEE Photon. Technol. Lett., vol. 18, pp. 2584-2586, 2006.

[11] Vita P. Di. and Rossi U., "Backscattering measurements in optical fibers: Separation of power decay from imperfection contribution," Electron. Lett., vol. 15, pp. 467-469, 1979.

[12] Brinkmeyer E., "Analysis of the backscattering method for single-mode optical fibers," J. Opt. Soc. Amer., vol. 70, pp. 1010-1012, 1980.

[13] O'Sullivan M. S. and Ferner J., "Interpretation of SM fiber OTDR signatures," in Proc. SPIE'86 Optic. Testing Metrology, vol. 661, pp. 171-176, 1986.

[14] Tsujikawa K., Ohashi M., Shiraki K., and Tateda M., "Scattering property of F and $\mathrm{GeO}_{2}$ codoped silica glasses," Electron. Lett., vol.30, pp. 351-352, 1994.

[15] Rossaro A., Schiano M., Tambosso T. and D'Alessandro D., “Spatially resolved chromatic dispersion measurement by a bidirectional OTDR technique," IEEE J. Select. Topics Quantum Electron., vol. 7, pp. 475-483, 2001.

[16] ITU-T Recommendation COM15-55-E, Dec. 1997.

[17] Pask C., "Physical interpretation of Peterman's strange spot size for single-mode fibers," Electron. Lett., vol. 20, pp. 144-145, 1984.

[18] Shibata N., Kawachi M., and Edahira T., "Optical loss characteristics of high- $\mathrm{GeO}_{2}$ content silica fibers," IECE(J) Trans., vol. E63, pp. 837-841, 1980.

[19] Marcuse D., “Loss analysis of single mode fiber splice," Bell Syst. Tech. J., vol. 56, pp. 703-718, 1977. 
[20] Takachio N., Suzuki H., Masuda H., and Koga M., “32x10 Gb/s distributed Raman amplification transmission with $50-\mathrm{GHz}$ channel spacing in the zero-dispersion region over $640 \mathrm{~km}$ of $1.55-\mu \mathrm{m}$ dispersion- shifted fiber," presented at the Optical Fiber Communication Conf. and Int. Conf. Integrated Optics Optical Fiber Communication (OFC/ IOOC'99), Postdeadline Paper PD9.1, 1999.

[21] Nissov M., Davidson C. R., Rottwitt K., Menges R., Corbett P. C., Innis D., and Bergano N. S., "100 Gb/s (10x10 Gb/s) WDM transmission over $7200 \mathrm{~km}$ using distributed Raman amplification," in Proc. Eur. Conf. Opt. Commun. (ECOC), Edinburgh, Scotland, pp. 9-12, 1997.

[22] Agrawal G. P., Nonlinear Fiber Optics, 3rd ed. New York: Academic Press, 1995.

[23] Shibata N., Horiguchi M., and Edahiro T., "Raman spectra of binary high-silica glasses and fibers containing $\mathrm{GeO}_{2}, \mathrm{P}_{2} \mathrm{O}_{5}$ and $\mathrm{B}_{2} \mathrm{O}_{3}$," J. Non-Cryst. Solids, vol. 45, pp. 115-126, 1981.

[24] Nakashima T., Seikai S., and Nakazawa M., "Dependence of Raman gain on relative index difference for $\mathrm{GeO}_{2}$-doped single-mode fibers," Opt. Lett., vol. 10, pp. 420-422, 1985. 
